\title{
Gating of a molecular transistor: Electrostatic and Conformational
}

\author{
Avik W. Ghosh, Titash Rakshit and Supriyo Datta \\ School of Electrical and Computer Engineering, Purdue University, W. Lafayette, IN 47907
}

(Dated: October 29, 2018)

\begin{abstract}
We derive a general result that can be used to evaluate and compare the transconductance of different field-effect mechanisms in molecular transistors, both electrostatic and conformational. The electrostatic component leads to the well-known thermal limit in the absence of tunneling. We show that in a standard three-terminal geometry and in the absence of strong electron-phonon coupling, the conformational component can lead to significant advantages only if the molecular dipole moment $\mu$ is comparable to $e t_{\mathrm{ox}}, t_{\mathrm{ox}}$ being the thickness of the oxide. Surprisingly this conclusion is independent of the "softness" of the conformational modes involved, or other geometrical factors. Detailed numerical results for specific examples are presented in support of the analytical results.
\end{abstract}

PACS numbers: PACS numbers: 85.65.+h, 73.23.-b,31.15.Ar

Device miniaturization is progressively heading towards solid state electronic components that are molecular in nature 1, 2]. Molecular transistors are of great current interest from both basic and applied points of view. Despite theoretical proposals 3, 4, 5] and experimental reports [6, 7, 8] of three-terminal molecular devices, their general physical principles are not yet well understood. In a standard silicon MOSFET the gate modulates the current by controlling the channel charge through its electrostatic potential. Good transistor action requires that this channel potential respond much more to the gate than to the drain, implying an oxide thickness $t_{\mathrm{ox}}$ that is much smaller than the channel length $L$ (Fig. 17). A molecular transistor operating on electrostatic principles ought to have the same design limitation, so that even nominal gate control in a $10 \AA$ molecule such as phenyl dithiol (PDT) 1] demands an oxide that is prohibitively thin [9]. It is therefore natural to investigate alternate principles of molecular transistor action, such as by utilizing conformational degrees of freedom [4]. For instance, a gate field coupled with a molecular dipole moment $\vec{\mu}$ could cut off the current by tilting it away from a contact (Fig. 1b), or by twisting one part relative to the other, breaking its conjugation [10, 11] (Fig. [1). Such a mechanism could respond more strongly to the gate than the drain if $\vec{\mu}$ is engineered to lie along the source-drain field.

In this paper, we derive a general expression for the transconductance per unit current $g_{m} / I=$ $(1 / I)\left(\partial I / \partial V_{g}\right)\left(I\right.$ : current, $V_{g}$ : gate voltage) 12] for different transistor mechanisms, both electrostatic and conformational [15]. For a large separation of time scales between electronic and vibronic modes, as is typical in room temperature molecular conductors [16], the electrostatic and conformational transconductances are additive, with a well-known electrostatic maximum:

$$
\left[g_{m}^{\mathrm{es}} / I\right]_{\max }=e \beta=(25 m V)^{-1}=40 \mathrm{~V}^{-1}
$$

( $\beta$ : inverse thermal energy), achieved only if $t_{\mathrm{ox}} \ll L$ and if there is negligible tailing in the density of states. In ad-

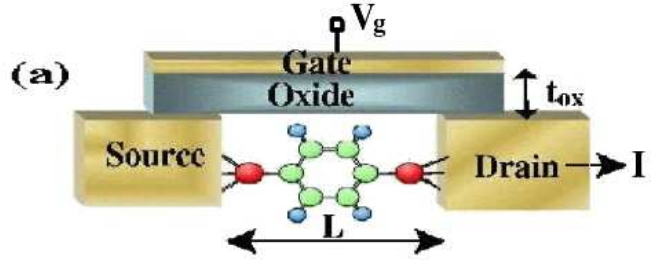

(b)

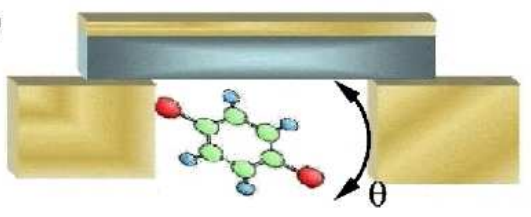

(c)

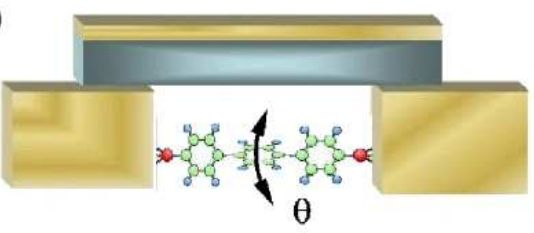

FIG. 1: In a standard transistor (a) the gate voltage $V_{g}$ controls the current $I$ by controlling the induced charge. But in conformational transistors the gate controls the current through the configurational parameter $\theta$. This could be either (b) through a variation in coupling with the contact, or (c) a variation in transmission through the molecule itself.

dition, we find a fundamental conformational maximum, independent of modal stiffness or molecular geometry:

$$
\left[g_{m}^{\text {conf }} / I\right]_{\max }=e \beta\left(\mu / e t_{\mathrm{ox}}\right) .
$$

These simple expressions for gate control are later corroborated with detailed numerical results that selfconsistently combine a semi-empirical description of the device Hamiltonian with a nonequilibrium Green's function (NEGF) description of transport. Based on these results, it seems that good molecular transistor action in a standard MOS geometry with a modest gate insulator thickness would require degenerately doped semiconducting contacts, along with the incorporation of a molecular dipole dipole aligned along the source-drain direction and large enough to overcome any room-temperature thermal 
averaging over conformations, ie, larger than $e t_{\mathrm{ox}}$.

We assume above that conducting and non-conducting molecular states lie in the same valley in the conformational potential landscape. The gate voltage shifts the minimum within this valley but thermal excitations lead to a statistical average over the entire valley, making it difficult to change the current any faster than the fundamental limit implied by Eq. 2 One could get around this limit if the conducting and non-conducting molecular states belong to distinct metastable valleys [4], such that thermal averaging takes place only over one valley or the other. The role of the gate then is to shift the molecule from one valley to the other through a large impulsive force. Such a mechanism, however, is very different from the operating principles of present day MOS devices.

We will now discuss these issues quantitatively by deriving Eq. 5 for $g_{m} / I$ and evaluating its electrostatic and conformational components for specific illustrative examples, with detailed numerical calculations for support.

Transconductance equation. A source-drain bias $V_{d}$ splits the contact chemical potentials $\mu_{1,2}$ by the applied voltage. The molecular I-V is obtained from Landauer theory [13], which can be recast in the less familiar form:

$$
\begin{aligned}
I & =\frac{2 e}{h} \int_{\mu_{1}}^{\mu_{2}} d E\langle\tilde{T}(E)\rangle ; \\
\tilde{T}(E) & =T(E) \otimes F_{T}(E) .
\end{aligned}
$$

Thermal broadening appears 14 as a convolution $\otimes$ of the transmission $T(E)$ at zero lead temperature with the thermal broadening function $F_{T}(E)=-\partial f(E) / \partial E$, $f(E)=1 /[\exp (E \beta)+1] .\langle\ldots\rangle$ gives a thermal average over various molecular configurations $\{x\}$ with probabilities set by the conformational potential energies $U(\{x\})$ :

$$
\langle\tilde{T}(E)\rangle=\frac{\sum_{i} \tilde{T}\left(E ; x_{i}\right) w_{i}}{\sum_{i} w_{i}}, \quad w_{i}=\exp \left[-\beta U\left(x_{i}\right)\right]
$$

$x_{i}$ denoting the coordinate of the ith configuration. From Eq. 3. $I=(2 e / h)\left(\mu_{1}-\mu_{2}\right)\left\langle\tilde{T}_{0}\right\rangle, \tilde{T}_{0}$ being the average value of $\tilde{T}(E)$ over the energy range $\mu_{1}<E<\mu_{2}$. From Eq. [4 straightforward algebra gives $g_{m}=g_{m}^{\text {es }}+g_{m}^{\text {conf }}$, where

$$
\begin{aligned}
\frac{g_{m}^{\text {es }}}{I} & =\frac{1}{\left\langle\tilde{T}_{0}\right\rangle}\left\langle\frac{\partial \tilde{T}_{0}}{\partial V_{g}}\right\rangle, \\
\frac{g_{m}^{\text {conf }}}{I} & =\frac{\beta}{\left\langle\tilde{T}_{0}\right\rangle}\left[\left\langle\tilde{T}_{0}\right\rangle\left\langle\frac{\partial U}{\partial V_{g}}\right\rangle-\left\langle\tilde{T}_{0} \frac{\partial U}{\partial V_{g}}\right\rangle\right] .
\end{aligned}
$$

The transconductance has two additive contributions: an electrostatic term describing how the gate controls the channel charge, and a conformational term describing how the gate controls the transmission by deforming the molecule [16. We now discuss these terms one by one.

Electrostatic control. Electrostatically the gate modulates the self-consistent potential, effectively moving the molecular energy levels relative to the contacts. A complete treatment of this effect requires a self-consistent solution of the Schrödinger-Poisson equations. However, one can get a qualitative description by assuming that a gate bias rigidly shifts the energy levels and hence the transmission: $T\left(E, V_{g}\right) \approx T\left(E-\gamma e V_{g}\right)$. The parameter $\gamma$ tells us the average molecular potential $V_{m}$ in response to a change in gate bias: $\gamma \equiv \partial V_{m} / \partial V_{g}$. Using Eq. 5 a

$$
\frac{g_{m}^{\mathrm{es}}}{I}=\frac{e \gamma}{\left\langle\tilde{T}_{0}\right\rangle}\left\langle\frac{\partial \tilde{T}_{0}}{\partial E}\right\rangle
$$

$\gamma \sim C_{G} /\left(C_{G}+C_{S}+C_{D}+C_{Q}\right)$, where $C_{G, S, D}$ represent the effective capacitances coupling the molecule to the gate, source and drain respectively, while $C_{Q}$ is the quantum capacitance proportional to the molecular density of states [17]. One could view $C_{G, S, D}$ as a representation of Poisson's equation, and $C_{Q}$ as a linearized representation of Schrödinger's equation. Under OFF conditions $C_{Q}$ is negligible and $\gamma$ can be made close to one in a welldesigned FET by making $t_{\mathrm{ox}} \ll L$, so that $C_{G} \gg C_{S, D}$. However, as we noted earlier, this becomes increasingly difficult as we try to engineer ultrasmall devices that are only tens of atoms long ( $L \sim 1-5 \mathrm{~nm})$. The electrostatic parameter $\gamma$ is then severely reduced, motivating us to look for non-electrostatic mechanisms for control. Note that the electrostatic restriction on $\gamma$ is fundamental, and may not be easy to handle without using large dielectric constant insulators with a small effective thickness.

Eq. [6] also elucidates the role of a sharp transition in $\tilde{T}_{0}$ as a function of $E$ in realizing good electrostatic control. A metallic conductor with constant $\tilde{T}_{0}(E)$ cannot be used to build an electrostatic switch. Semiconductors by contrast have a band-edge where $\tilde{T}_{0}(E)$ drops to zero over a very small energy range $\Delta E$, so that from Eq. [6]

$$
g_{m}^{\mathrm{es}} / I \approx e \gamma / \Delta E .
$$

Since $\tilde{T}_{0}(E)$ represents a convolution of $T(E)$ and $F_{T}(E)$, the minimum value of $\Delta E$ equals that for $F_{T}(E)$, which is $\sim 1 / \beta$. This leads to the well-known upper limit of $e \beta$ for $g_{m}^{\text {es }} / I$, corresponding to $\gamma=1$ and $\Delta E=1 / \beta$. An important point to note is that increased tunneling in nanodevices can make $T(E)$ non-zero below bandgap, with $\Delta E \gg 1 / \beta$. This condition is made worse by the use of metallic (rather than semiconducting) source and drain regions which is common for molecular devices 1, 2]. In view of recent progress in growing molecules on silicon surfaces [18], using doped semiconducting contacts seems a realizable and highly desirable ideal.

Conformational control. The conformational mechanism (Eq. 5b) operates by changing the relative energies $U$ for different molecular configurations $\left\{x_{i}\right\}$. As expected, we get zero transconductance if $\tilde{T}_{0}$ is independent of the configuration $\left\{x_{i}\right\}$, or its variation is uncorrelated with $\partial U / \partial V_{g}$. But if $\tilde{T}_{0}$ and $\partial U / \partial V_{g}$ are negatively correlated such that configurations with larger transmission 
$\tilde{T}_{0}$ have their energies $U$ reduced by the gate voltage $V_{g}$ (and hence made more likely) then the current increases, with a positive $g_{m}^{\text {conf }} / I$. To estimate $g_{m}^{\text {conf }} / I$, we write:

$$
U\left(\theta ; V_{d} ; V_{g}\right)=U_{0}(\theta)-\left(\mu V_{g} / t_{\text {ox }}\right) \sin \theta-\left(\mu V_{d} / L\right) \cos \theta,
$$

where $\theta$ is the angle between the molecular dipole $\vec{\mu}$ and the source-drain field $V_{d} / L$. Aligning the dipole along the latter eliminates its torque [19], giving the gate field $V_{g} / t_{\mathrm{ox}}$ an obvious superiority. Using Eq. 8 in Eq. $5 \mathrm{~b}$

$$
\frac{g_{m}^{\mathrm{conf}}}{I}=-\beta \frac{\mu}{t_{\mathrm{ox}}}\left[\frac{\left\langle\tilde{T}_{0}\right\rangle\langle\sin \theta\rangle-\left\langle\tilde{T}_{0} \sin \theta\right\rangle}{\left\langle\tilde{T}_{0}\right\rangle}\right] .
$$

The quantity within parantheses has a maximum value of one, indicating that the maximum conformational transconductance is given by $\mu \beta / t_{\mathrm{ox}}$ as stated earlier (see Eq. (2). If $\mu$ is comparable to $e t_{\mathrm{ox}}$, then it is indeed possible for this mechanism to provide respectable levels of control. For a $10 \AA$ oxide, $\mu / e t_{\text {ox }} \sim 0.15$ for an aromatic molecule with one redox $\mathrm{NO}_{2}$ sidegroup per benzene ring. Although it is hard to squeeze in any more dipolar subgroups per ring, one could incorporate large effective dipoles external to the molecule, using a strong piezoelectric gate-molecular coupling, for instance [7]. In any case, a conformational transconductance that is, say, a tenth of $e \beta$ may still be useful in view of the difficulties with the electrostatic mechanism discussed earlier.

Significantly, the upper limit on the conformational transconductance (Eq. 2) is unaffected by the 'softness' of the modes described by $U_{0}(\theta)$ in Eq. 8 For the bending and twisting configurations in Figs \(b,c), we write:

$$
\begin{aligned}
& U_{0}(\theta)=U_{0}^{\text {bend }}\left(\theta-\theta_{0}\right)^{2} / 2, \\
& U_{0}(\theta)=U_{0}^{\text {twist }}(1-\cos 2 \theta) / 2 .
\end{aligned}
$$

Twisting a molecular bond is much easier than bending it $\left(U_{0}^{\text {bend }} \sim 2 \mathrm{eV}[20], U_{0}^{\text {twist }} \sim 0.03 \mathrm{eV}[21]\right)$; however, twisting also makes thermal averaging more significant, so that no advantage is gained as far as $g_{m}$ is concerned.

The quantity inside parentheses in Eq. 9 can ideally have a maximum value of one if $\tilde{T}_{0}$ is very sharply varying. In practice it is smaller, depending on the variation of $\tilde{T}_{0}$ with $\theta$. As an example, we model a self-assembled PDT monolayer (Fig. \) chemisorbed on a $\mathrm{Au}(111)$ substrate, varying the tilt angle from default $\left(\theta_{0} \sim 20^{\circ}\right)$ to upright position and increasing the sulphur-gold coupling exponentially. Although accidental symmetries at specific angles of the highly directional orbital wavefunctions quench the overlap [11, 22], such orientational effects wash out on averaging over various possible positions of the sulphur and gold surface atoms in the upright configuration. The dominant angular dependence in $T_{0}$ then arises from the overlap between sulphur and gold $r a$ dial wavefunctions: $T_{0}(\theta) \propto \exp \left[-2 Z L(1-\cos \theta) / n a_{0}\right]$, $(Z=3$ : screened nuclear charge, $n$ : principal quantum number of sulphur, $\left.a_{0}=0.529 \AA\right)$. This gives a correlation in Eq. 9 of $\sim 0.1$ at room temperature $(\mu \approx 8$ Debye, $\left.V_{g} / t_{\mathrm{ox}}=1 \mathrm{~V} / \mathrm{nm}\right)$. This variation can be made to approach a delta-function by increasing $L$, giving a stronger correlation. Similarly for the rotational configuration in Fig. 11: we find $T_{0}(\theta) \propto \cos ^{4} \theta$ [23], leading to a configurational average $\sim 0.3$. The correlation depends on the variation $T_{0}(\theta)$, but also on the weighting factors which in turn depend on the bond stiffness, temperature, gate field and dipole moment.

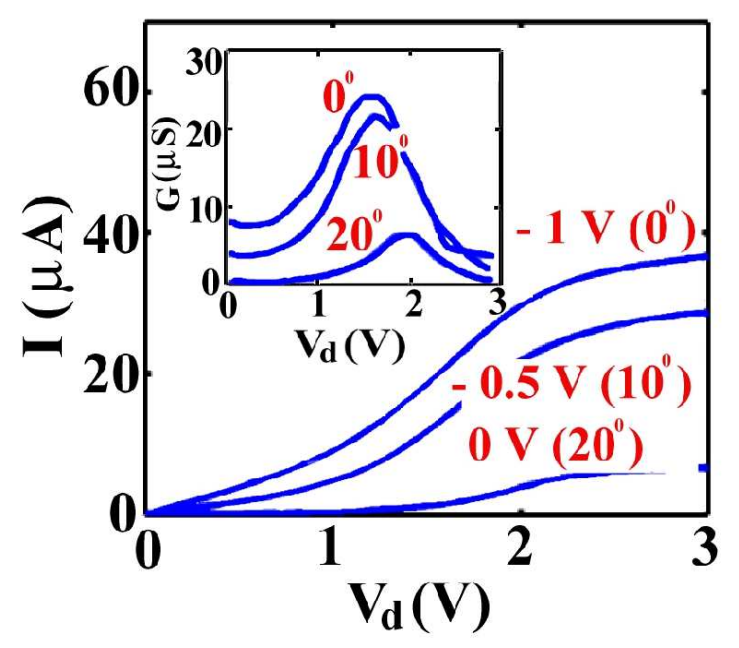

FIG. 2: The I-V for PDT at a given angle saturates on crossing the highest occupied molecular orbital (HOMO) level, increasing with decreasing tilt angle. The electrostatic gate influence (inset) causes a shift in the conductance peak towards lower voltages for p-type (HOMO) conduction.

Numerical Results. We now present detailed calculations of the I-V for the structures in Figs. 10 15. The transmission at a given angle is calculated using the nonequilibrium Green's function (NEGF) formalism 24] with an extended Hückel Hamiltonian $H$. We solve

$$
\begin{aligned}
G(E) & =\left(E S-H-U(\rho)-\Sigma_{1}-\Sigma_{2}\right)^{-1}, \\
\rho & =\int_{-\infty}^{\infty}\left(f_{1} G \Gamma_{1} G^{\dagger}+f_{2} G \Gamma_{2} G^{\dagger}\right) d E / 2 \pi, \\
T & =\operatorname{trace}\left(\Gamma_{1} G \Gamma_{2} G^{\dagger}\right),
\end{aligned}
$$

self-consistently $\left(S\right.$ : overlap matrix, $\left.f_{1,2}=f\left(E-\mu_{1,2}\right)\right)$. An ideal $\mathrm{Au}(111)$ surface geometry 24] is used to calculate the contact self-energies $\Sigma_{1,2}$ and level broadenings $\Gamma_{1,2}$. The self-consistent potential $U(\rho)$ is obtained by solving Laplace's equation for the device geometry with a $10 \AA$ oxide and a Hubbard-type electron-electron term.

Fig. 2 shows the zero-temperature I-V for a PDT relay for various tilt angles. The current tends to saturate on crossing the HOMO level, and increases with decreasing tilt (increasing $V_{g}$ ). In addition, there is a conventional electrostatic gate influence [9]. For a p-type (HOMO) conduction, a negative gate bias raises the molecular lev- 
els relative to the contacts, producing a lateral shift in the conductance $\left(G=\partial I / \partial V_{d}\right)$ towards lower $\left|V_{d}\right|$ (inset).

Fig. [3] shows the three-terminal I-V of the Tour-Reed molecule 25] containing a nitroamine redox group at (a) $0 \mathrm{~K}$ and (b) $300 \mathrm{~K}$ [26]. $\quad E_{F}$ is assumed to lie in the HOMO tail, giving an ohmic I-V at low $V_{d}$. The impedance is compromised by the poor electrostatics due to the oxide thickness and MIGS, as discussed earlier. The low-temperature $g_{m} / I$ is impressive due to the low torsion constant and the large dipole, a mere $400 \mathrm{mV}$ gate bias reducing the current two hundred times. At room temperature, however, the molecule samples a wide range of angles, reducing $g_{m} / I$ substantially. The gatemodulation continues to be observable, but the current is a lot harder to switch off. A lower torsion constant gives better orate control hut increases thermal effects as well.

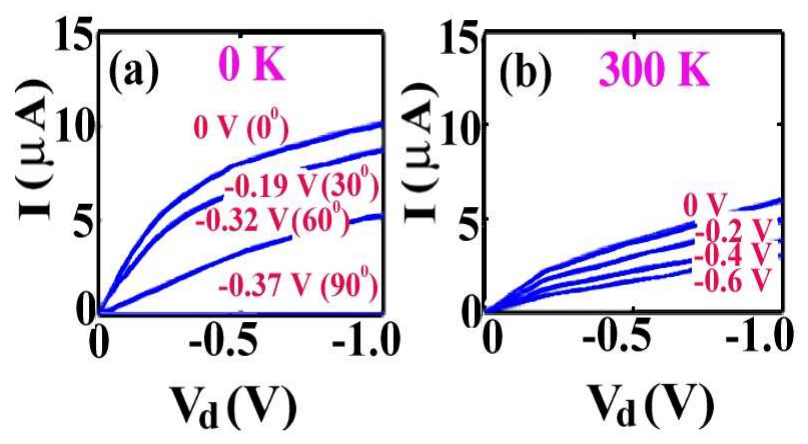

FIG. 3: I-V of the Tour-Reed molecule 25] for various gate voltages at (a) $0 \mathrm{~K}$ and (b) $300 \mathrm{~K}, E_{F}$ lying in the tail of the HOMO level. At $300 \mathrm{~K}$ there is some gate modulation, although it is hard to completely switch the current off.

The performance of a transistor also depends on its operating speed. While complicated isomeric cis-trans rotations tend to be slow, bond rotations are much faster ( 10-100 GHz), and need to be damped out without consuming too much power. Image forces and Van der Waals interactions with electrodes [27] as well as steric and hydrogen bonding interactions between molecules 5 in a mixed monolayer could damp out such oscillations.

We have shown that conformational transitions can aid electrostatic gate control significantly if we could engineer a large molecular dipole along a suitable direction. Ways around such design restrictions require going beyond Eq. 8 employing other kinds of electromechanical gate-molecular coupling (piezoelectric for example), or using non-traditional, bistable potentials with large impulsive gate voltages [4]. Eq. 5 gives us a general way to quantitively compare the transconductance of these various field-effect mechanisms in molecular transistors.

We thank P. Damle, D. Monroe, P. Solomon and M. Lundstrom for useful discussions, and T. Raza for the image in the table of contents. This work is supported by the US ARO and the Semiconductor Technology Focus Center on Materials, Structures and Devices.
[1] M. A. Reed et al., Science 278, 252 (1997).

[2] C. Dekker, Phys. Today 52, 22 (1999); B. Chen et al., J. Phys. Chem. B 103, 4447 (1999); C. Kergueris et al., Phys. Rev. B 59, 12505 (1999); J. Reichert et al., Phys. Rev. Lett. 88, 176804 (2002).

[3] M. Di Ventra, S. T. Pantelides and N. D. Lang, Appl. Phys. Lett. 76, 3448 (2000); E. G. Emberly and G. Kirczenow, Phys. Rev. B 62, 10451 (2000).

[4] Y. Wada, Proc. IEEE 89, 1147 (2001); P. von Allmen and K. Hess, Phys. Rev. B 52, 5243 (1995).

[5] D. GoldhaberGordon et al., Proc. IEEE 85, 521 (1997).

[6] H. Park et al., Nature 407, 57 (2000).

[7] C. Joachim and J. K. Gimzewski, Chem. Phys. Lett. 265, 353 (1997).

[8] J-O Lee et al., Nano Lett. 3, 113 (2003); C. R. Kagan et al., ibid, pp. 119.

[9] P. Damle, T. Rakshit, M. Paulsson and S. Datta, cond-mat/0206328

[10] The end rings are tethered to the contacts and are relatively immobile. The relative twist can be further enhanced using a four ring extension, with diametrically opposite dipoles on the central rings rotating in opposite directions in the gate field.

[11] M. P. Samanta, W. Tian, S. Datta, J. I. Henderson and C. P. Kubiak, Phys. Rev. B 53, R7626 (1995); M. Magoga and C. Joachim, Phys. Rev. B 59, 16011 (1999); C. Zhou, Ph.D. thesis, Yale University (1999).

[12] We use $g_{m} / I$ rather than $g_{m}$ to ensure that this 'figure of merit' is a measure of the effectiveness of the mechanism rather than of the number of molecules involved.

[13] S. Datta, "Electronic Transport in Mesoscopic Systems", Cambridge University Press (1995).

[14] P. F. Bagwell and T. P. Orlando, Phys. Rev. B 40, 1456 (1989)

[15] We are not considering resonant or single-electron tunneling devices. The former can be analyzed using Eqs. 345 but the latter needs a many-particle description.

[16] The additivity of the two contributions arises from the widely different time-scales between electronic $(\sim 10$ $\mathrm{THz})$ and conformational $(\sim 10 \mathrm{GHz})$ modes. This causes a relatively weak influence of vibrations on the $\mathrm{I}-\mathrm{V}$ even at high bias (see for example, M. Di Ventra et al., Phys. Rev. Lett. 88, 046801 (2002)).

[17] S. Luryi, Appl. Phys. Lett. 52, 501 (1988).

[18] R. A. Wolkow, Jpn. J. Appl. Phys. 1 40, 4378 (2001): N. P. Guisinger et al., Nano Letters 4, 55 (2004).

[19] Eliminating source-drain torque may require a doublegated device (P. Solomon, private communications).

[20] H. H. Jung et al., Langmuir 15, 1147 (1999).

[21] K. Saito et al., J. Phys. Cond. Matt. 7, 8919 (1995).

[22] A. M. Bratkovsky and P. E. Kornilovich, cond-mat/0204597

[23] Including multiple coherent reflections between rings makes $\tilde{T}_{0}(\theta)$ more complicated (see [13], Eq. 3.2.4).

[24] P. S. Damle, A. W. Ghosh and S. Datta, Phys. Rev. B 64, 201403(R) (2001).

[25] J. Chen et al., Science 286, 1550 (1999).

[26] Experimentally these molecules show negative differential resistance [25], but the mechanism is unclear and does not follow from the formulation used here.

[27] M. Dequesnes et al., Nanotechnology 13, 120 (2002). 